\title{
NUEVAS Y VIEJAS FORMAS DE REGULACIÓN DEL SISTEMA EDUCATIVO EN ARGENTINA
}

NOVAS E VELHAS FORMAS DE REGULAÇÃO DO SISTEMA EDUCATIVO NA ARGENTINA

\author{
NEW AND OLD FORM OF REGULATION \\ OF THE EDUCATIONAL SYSTEM IN ARGENTINA
}
DE NOUVELLES ET VIEILLES FORMES DE REGULATION DU SYSTEME EDUCATIONNEL EN ARGENTINE

\author{
Myriam Feldfeber*
}

\section{RESUMEN}

En este trabajo analizamos las politicas destinadas a regular el sistema educativo a partir de la transferencia de servicios educativos a los estados sub-nacionales y la redefinición del rol histórico que el Estado Nacional asumió en materia educativa. Sostenemos que las políticas impulsadas desde el Estado Nacional han estado orientadas a dar respuesta a la crisis del modelo de regulación burocrático tradicional y a garantizar la gobernabilidad del sistema a través de la introducción de nuevas tecnologías de gestión y del desarrollo de políticas compensatorias al servicio de la reducción de la pobreza. Estas políticas se han propuesto como la única alternativa de construcción política viable para asentar el gobierno de la educación sobre nuevas bases.

Palabras-clave: Reforma educativa. Politicas educativas. Regulación. Gobierno.

* Profesora e investigadora de la Universidad de Buenos Aires - Argentina (mfeldfeb@filo.uba.ar).

Este artículo es una versión corregida del trabajo presentado en "The 2007 Meeting of the Latin American Studies Association, Montreal, Canadá, September 5-8, 2007”. Session: Regulación de las políticas educativas en América Latina. Chair: Dalila Andrade Oliveira. El trabajo integra el Proyecto de Investigación Ubacyt (2004-2007) "Las políticas de municipalización y autonomía escolar: regulación estatal, actores y procesos en el caso argentino". Instituto de Investigaciones en Ciencias de la Educación. Universidad de Buenos Aires. Directora: Myriam Feldfeber. Agradezco los valiosos aportes de Nora Gluz, investigadora del Proyecto Ubacyt y de la UNGS. 


\section{INTRODUCCIÓN}

Después de dos décadas de aplicación de políticas de ajuste estructural formuladas de acuerdo con los postulados del denominado Consenso de Washington, los resultados son más que elocuentes. América Latina es una región rica en la que millones de personas viven en condición de pobreza (la mayoría son niñas y niños) y es una de las regiones más desiguales del planeta en términos de distribución del ingreso, ubicándose incluso por detrás de regiones con índices de desarrollo mucho más bajos. Argentina no ha sido una excepción en este sentido sino que, por el contrario, algunas de las reformas implementadas en nuestro país han sido paradigmáticas en la región.

En el marco de la aplicación de estas políticas, las orientaciones económicas neoliberales adoptadas desde comienzos de los '90, insertaron a la Argentina de un modo pasivo en el proceso mundial de globalización. Las decisiones del gobierno nacional, que modificaron de manera radical los vínculos entre el Estado y la sociedad, fueron el resultado de una trama compleja de condiciones socio-estructurales y de relaciones socio-políticas. El neoliberalismo como propuesta de política económica, contó con el apoyo de los principales sectores propietarios del país que desde hacía muchos años criticaban el dirigismo estatal (SIDICARO, 2003).

En materia educativa, la reforma implementada durante las últimas décadas también puede ser analizada como una respuesta frente a la crisis de la matriz estadocéntrica (CAVAROZZI, 1992) que caracterizó la institucionalización y expansión del sistema educativo en Argentina. El paradigma característico del Estado burocrático fue desplazado por el paradigma gerencialista (GARCÍA DELGADO, 1996), vinculado a la idea de una política pública eficiente, eficaz y evaluable en términos de resultados.

En este trabajo analizamos las políticas destinadas a regular el sistema educativo a partir de la transferencia de servicios educativos a los estados sub-nacionales y la redefinición del rol histórico que el Estado Nacional asumió en materia educativa. Sostenemos que las políticas impulsadas desde el Estado Nacional han estado orientadas a dar respuesta a la crisis del modelo de regulación burocrático tradicional y a garantizar la gobernabilidad del sistema a través de la introducción de nuevas tecnologías de gestión y del desarrollo de políticas compensatorias al servicio de la reducción de la pobreza. Estas políticas se han propuesto como la única alternativa de construcción política viable para asentar el gobierno de la educación sobre nuevas bases.

La discusión sobre los nuevos modos de regulación de las políticas educativas (BARROSO, 2004; BOLIVAR, 2004) adquiere sentido a partir del proceso de reforma del Estado y los nuevos vínculos que se establecen entre Estado y sociedad. La experiencia privatizadora en América Latina ha generado una nutrida literatura sobre el tema, que refiere al rol regulador del Estado y a la regulación como problema teórico. Otra parte de los trabajos están dedicados a analizar técnicamente los problemas relacionados con la regulación en sectores específicos de servicios (OSZLAK, 2004). 
Sin embargo, el problema de la regulación en el campo educativo no puede enfocarse desde la perspectiva de la regulación de los servicios públicos privatizados.

Barroso (2005) propone un modelo interpretativo que pueda dar cuenta de los diferentes contextos disciplinares y lingüísticos que otorgan sentido a los nuevos modos de regulación de la política educativa. Señala que en un sistema social complejo como el sistema educativo existe una pluralidad de finalidades y modalidades de regulación en función de la diversidad de actores involucrados, de sus posiciones, de sus intereses y estrategias. En los sistemas públicos el Estado constituye una fuente esencial de regulación, aunque no es la única. La regulación del sistema educativo resulta de una regulación de las regulaciones más que del control directo de aplicación de una regla sobre la acción de los regulados.

\section{LA REGULACIÓN COMO PROBLEMA DE GOBIERNO EN EL MARCO DE UN PAÍS FEDERAL}

La distribución y articulación de responsabilidades, obligaciones y recursos entre el Estado Nacional y los Estados Provinciales para garantizar el derecho a la educación constituyó una fuente de conflictos desde los orígenes del sistema educativo argentino. La reconstrucción histórica de las políticas educativas en Argentina nos permite dar cuenta del pasaje de un sistema educativo creado y regulado desde el Estado Nacional, organizado en forma unificada y centralizado bajo pautas homogéneas de funcionamiento en el ámbito público, hacia un sistema educativo formalmente "descentralizado", diversificado y fragmentado pero bajo nuevas formas de regulación y control por parte del Estado Nacional.

Hasta mediados de siglo XX el gobierno nacional tuvo un papel hegemónico. El Estado nacional ejerció su rol protagónico mediante la creación de escuelas, la prestación del servicio educativo, el financiamiento y el apoyo técnico. A mediados de siglo comenzaron a desarrollarse dos procesos que redefinieron su rol: el sector privado fue adquiriendo cada vez mayor autonomía -hecho que se legitimó e impulsó a través de la legislacióny las escuelas nacionales se transfirieron a las provincias (PAVIGLIANITI, 1991).

La transferencia de establecimientos nacionales a las provincias para los niveles inicial y primario fue impulsada desde el Estado Nacional a partir del año 1956, y finalizó durante la última Dictadura Militar (1976-1983) cuando se transfirieron 6.779 escuelas sin financiamiento por parte del gobierno nacional, ni aumento de la coparticipación federal de impuestos. Esta política, impulsada por Ministerio de Economía, buscaba la reducción del déficit fiscal, aún cuando formalmente la transferencia se fundamentó en el respeto del federalismo y en el artículo 5 de la Constitución Nacional que otorga a las provincias la responsabilidad de garantizar la educación primaria (BRAVO, 1994).

En un primer momento, la política de transferencia trajo como consecuencia el cierre de establecimientos educativos en diversas provincias que carecían de los recursos para 
afrontar los gastos. Posteriormente, al no establecerse mecanismos de coordinación y al no jugar el Consejo Federal de Cultura y Educación ${ }^{1}$ un rol decisivo, se verificó una gran anarquía, fundamentalmente en el plano del curriculum y de las condiciones laborales de los docentes. Una de las consecuencias más significativas de la transferencia fue la aceleración del proceso de segmentación interna del sistema educativo nacional (BRASLAVSKY, 1983). ${ }^{2}$

Este proceso culminó durante la primera presidencia de Carlos Menem cuando se sancionó la Ley de Transferencia de Establecimientos de Nivel Medio y Superior No Universitario (1991). También se transfirieron las escuelas privadas subvencionadas por el gobierno nacional.

En general, los trabajos de investigación señalan que la transferencia constituyó básicamente un desplazamiento de responsabilidades financieras y administrativas desde el nivel nacional a las provincias y la Ciudad de Buenos Aires. Implicó una mayor carga presupuestaria para las administraciones provinciales y la Ciudad de Buenos Aires (y un alivio fiscal para el nivel nacional), y en muchos casos contribuyó al deterioro de la calidad de la prestación del servicio educativo (BRAVO, 1994; KISILEVSKY, 1998; ECHART, 1998; REPETTO, 2001). Es por ello que se trató de un proceso de transferencia y no de descentralización entendida como delegación de poder. Novick de Senén Gonzalez (1994) señala que a diferencia de otros casos - como el mexicano-, no se previeron modelos alternativos o transitorios de gestión de los servicios transferidos. Ello llevó a situaciones muy heterogéneas debido a que las provincias presentan profundas diferencias en cuanto a su capacidad institucional y administrativa. Sólo unas pocas lograron integrar en forma armónica y sin conflictos la totalidad de los servicios transferidos al sistema provincial. ${ }^{3}$

En el caso específico de las Instituciones de Formación Docente, estas fueron transferidas sin que existiese un debate acerca de la conveniencia de que la formación-que se encontraba en gran parte bajo la dependencia del gobierno central- permaneciese en su órbita, lo que estaba previsto en la Ley de Transferencia que obligaba a transferir las escuelas de nivel medio pero no las instituciones de nivel superior no universitario (FELDFEBER; IVANIER, 2003).

Es importante destacar el papel que jugaron los organismos financieros internacionales, como el BID y el Banco Mundial, a través de los créditos que otorgaron al país para llevar a cabo estas reformas. Estos créditos estuvieron orientados y supeditados a la creación o al fortalecimiento de esos mecanismos, y a la contratación de muchos de los técnicos que los diseñaron y que apoyaron su implementación. El papel activo que tuvieron estos organismos financieros, así como otros de carácter "técnico" como la Cepal y la Unesco ${ }^{4}$ en la promoción de estos principios explica que se encuentren importantes similitudes con las reformas que se implementaron en otros países de la región.

Una vez transferidas las instituciones, las principales orientaciones de la política educativa respondieron a las denominadas "reformas del Estado de segunda generación", 
vinculadas con el establecimiento de un nuevo paradigma de la gestión pública bajo la influencia de la lógica empresarial y de los cambios en el modelo productivo y en el mercado de trabajo. A diferencia del caso brasilero, las discusiones en torno sobre la gestión democrática de la educación estuvieron ausentes en la agenda educativa definida desde las esferas oficiales.

La transformación encarada en forma compulsiva por el gobierno nacional a partir de la sanción de las Ley Federal de Educación en el año 1993 incluyó dispositivos que buscaron establecer nuevos mecanismos de regulación por parte del gobierno nacional que ya no tenía escuelas a su cargo. La legislación constituyó uno de los instrumentos privilegiados de política para direccionar esta nueva regulación educativa. ${ }^{6}$ Sin embargo, las transformaciones impulsadas a través de la legislación no han encontrado su correlato en los programas de gobierno para la implementación de los cambios legislativos.?

El Ministerio de Educación de la Nación intentó asumir un papel central a través de su influencia en la definición de los temas a incorporarse en la agenda de discusión y en los acuerdos del Consejo Federal de Cultura y Educación, y a través del manejo de recursos técnicos y financieros. La regulación del sistema se basó en el diseño de los Contenidos Básicos Comunes y nuevos modelos de gestión institucional, en el establecimiento del Sistema Nacional de Evaluación de la Calidad (SINEC) y del Sistema Nacional de Acreditación de los Institutos y de sus carreras, en la implementación de la Red Federal de Formación Docente Continua y en el desarrollo de políticas asistenciales focalizadas como el Plan Social Educativo (PSE).

En lo que respecta a los nuevos modelos de gestión institucional, el Ministerio "sin escuelas", enarboló un nuevo discurso basado en la autonomía institucional y en la responsabilización por los resultados educativos que se articuló con los viejos mecanismos de funcionamiento centralizados y burocráticos del sistema. Esto devino en la instalación de mecanismos de regulación central a la vez que se desreguló la responsabilidad por el sostenimiento directo del sistema y por sus resultados. Así, cuando se trató de implementar la reforma, el gobierno central presionó a las provincias para imponer sus criterios pero, en nombre del federalismo, éstas quedaron libradas a su suerte para garantizar el sostenimiento del sistema. El proceso de centralización de las decisiones se sustentó en la capacidad técnica y financiera del Ministerio de Cultura y Educación de la Nación puesta al servicio de la formulación de proyectos y demandas que invadieron las escuelas y establecieron los ritmos de la reforma.

Este recorrido pone de relieve que las preocupaciones por definir nuevos modos de gobierno de los sistemas educativos y de sus instituciones constituyeron una de las consecuencias más visibles de los procesos de reforma de la educación.

La búsqueda de nuevas formas de gobierno del sistema en el marco de la preocupación por la Gobernabilidad ${ }^{8}$ de los sistemas políticos constituyó una de las preocupaciones centrales a partir de la crisis del modelo de Estado de Bienestar. Con el retorno a 
la democracia en América Latina, las preocupaciones por la gobernabilidad comienzan a caracterizarse en términos de gobernabilidad democrática, pero sin despojarse de la connotación conservadora inicial vinculada con la necesidad de dar respuestas a las crisis generadas por la sobrecargas de demandas que el Estado no puede responder. Desde esta perspectiva, el único protagonista capaz de crear condiciones de gobernabilidad es la elite gobernante y las estrategias que se proponen frente a esta situación también conllevan una impronta conservadora: disciplinar a la sociedad a través de mecanismos ideológicos o coercitivos, a fin de limitar su capacidad de demanda. En los años '90, a partir de los documentos elaborados por los organismos internacionales, se retoma esta perspectiva en el marco de las discusiones sobre la gobernabilidad en América Latina. Otras perspectivas alertan sobre el uso restringido del concepto de gobernabilidad y plantean el debate en torno a la participación de los distintos actores sociales en función de la creación de las condiciones que hagan propicia la gobernabilidad democrática. Desde estas perspectivas, la gobernabilidad deja de ser un asunto de ingeniería en el ámbito de la cúpula del Estado para pasar a ser un proceso más complejo donde deben interactuar un conjunto de actores (FILMUS, 1995).

En este sentido, algunos trabajos centran la discusión en el Gobierno de la educación y se preguntan qué significa gobernar la educación y quién o quienes la gobiernan; preguntas que, situadas en el contexto del sistema educativo argentino deben tomar como punto de partida que la educación se parece cada vez menos a un sistema en términos de un centro que lo conduzca, los protagonistas son cada vez más numerosos y el juego que mantienen está cada vez más polarizado (TENTI FANFANI, 2004, p. 48).

Los debates en torno a los nuevos mecanismos para regular y gobernar los sistemas en el marco de los procesos de descentralización y desde el predominio del paradigma gerencialista se articularon en torno a la gestión de los centros y la autonomía escolar.

Entre los actuales modelos de regulación a través de las políticas de autonomía, podemos distinguir los modelos de regulación por los resultados de los modelos de regulación centrados en los procesos (MEURET, 2004). Asociada a la idea de autonomía escolar se encuentra la idea de que es necesario un nuevo tipo de vínculo entre las escuelas y el Estado. La idea de autonomía se construye con referencia al Estado y sus funciones en la regulación del proceso educativo. Existen al menos dos modelos de superación de la concepción racionalista-burocrática (BOLIVAR, 1996) o burocráticaprofesional (BARROSO, 2005) de los centros, donde mecanismos formales de control externos pueden regular la enseñanza y uniformar los resultados deseados. Uno de ellos ha hegemonizado las orientaciones de política de reforma de los '90, integrándose en lo que Roger Dale ha caracterizado como la agenda globalmente estructurada para la educación. Este modelo de regulación pos-burocrático se organiza en torno al Estado evaluador y a la lógica del cuasi-mercado, donde la mayor autonomía busca la diversificación de la oferta escolar y la promoción de la libertad de elección, acrecentando los 
mecanismos de evaluación externa (BARROSO, 2005). Este es el modelo que ha orientado las discusiones en torno a la autonomía escolar en la Argentina de los '90. El Programa Nueva Escuela para El Siglo XXI y el Proyecto de Escuelas Autogestionadas de San Luis constituyen ejemplos paradigmáticos de esta orientación (FELDFEBER, 2006). Otra alternativa de regulación se vincula con una concepción orgánica, donde la mayor autonomía busca incrementar la profesionalidad de los docentes y su compromiso con la mejora mediante formas comunitarias de participación (BOLIVAR, 1996).

En lo que respecta al sector docente, a diferencia de los modos de regulación típicamente burocráticos que apelaban a la docencia como categoría social homogénea, las nuevas formas post-burocráticas que buscan regular la formación y el trabajo de los docentes, se dirigen a éstos como individuos libres, que construyen su propia carrera y destino profesional. Se intenta pasar así del control burocrático característico de las sociedades disciplinarias (DELEUZE, 1991) a modelos que discursivamente proponen la autonomía profesional y la autorregulación, pero que condicionan dicha autonomía a través de medidas basadas en la lógica del mercado: redefinición de la carrera docente a partir de la flexibilización de las relaciones laborales, salario basado en el mérito, premio al desempeño, incentivos para atraer a "los mejores" a la profesión, evaluación basada en "reglas objetivas", mecanismos de acreditación y definición de estándares a nivel nacional e internacional (FELDFEBER, 2006). Estas nuevas formas de regulación han contribuido a precarizar las condiciones de trabajo de los docentes (OLIVEIRA, 2005).

\section{LAS POLÍTICAS DESTINADAS A LA REDUCCIÓN DE LA POBREZA EN UN CONTEXTO DE DESIGUALDAD}

Mientras que las recomendaciones de Política del Banco Mundial para el "sector educación" estuvieron orientadas en los años 80 por las propuestas de Ajuste Estructural, en los años 90 las propuestas del Banco enfatizaron la necesidad de que la educación contribuya a la reducción de la pobreza. ${ }^{9}$ En este sentido se propuso reasignar recursos públicos para atender la situación de los grupos específicos en situación de pobreza, los excluidos del modelo. La prioridad atribuida a la educación básica ha sido la gestión del trabajo y de la pobreza (OLIVEIRA, 2000).

La discusión sobre los nuevos modos de regulación de la política educativa no puede realizarse al margen de las condiciones en las que vive la población. La situación social en Argentina ha experimentado un notable deterioro en las últimas tres décadas. Mientras que la incidencia de la pobreza no llegaba al $10 \%$ de la población a mediados de la década del '70, llegó a superar el 40\% durante la hiperinflación de fines de la década del ' 80 , y el $50 \%$ luego de la crisis de 2001 . Si bien el porcentaje de la población que se encuentra en situación de pobreza ha disminuido sensiblemente a partir del notable crecimiento económico registrado en los últimos cinco años, aún se encuentra alrededor del 30\%, nivel muy superior al que Argentina había registrado históricamente. 
La distribución del ingreso también se ha deteriorado significativamente en el mismo período. En efecto, el coeficiente de Gini pasó de un nivel inferior a 0,40 a mediados de los '70 a 0,53 luego de la crisis de 2001. La recuperación del último lustro contribuyó a revertir la tendencia pero, a diferencia de lo ocurrido con la disminución de la pobreza, la mejora en la distribución no fue tan significativa, ubicándose el coeficiente de Gini en niveles de 0,50. La proporción entre el decil más rico y el más pobre de la población que era de 18 veces a principios de los '90, pasó a casi 40 veces luego de la crisis de 2001, para reducirse actualmente a 32 veces. De este modo, la notable recuperación de la economía no logró siquiera restablecer los patrones previos de distribución del ingreso.

El deterioro en las condiciones sociales se refleja tanto en las condiciones de permanencia en el sistema educativo como en los indicadores de rendimiento. En efecto, mientras que el índice de escolarización al inicio de la escolaridad primaria es cercano al $100 \%$, se registran importantes diferencias en la asistencia en función de la condición socio-económica de los alumnos.

Asistencia de la Población escolar por nivel educativo según condición de pobreza conglomerados urbanos, 2006

\begin{tabular}{|c|c|c|c|c|c|c|c|c|}
\hline \multirow{2}{*}{ Edad } & \multirow{2}{*}{$\begin{array}{c}\text { \% que } \\
\text { asiste }\end{array}$} & \multicolumn{3}{|c|}{ Pobre* $^{*}$\begin{tabular}{c}
\multirow{2}{*}{ \% que } \\
asiste
\end{tabular}} & \multicolumn{3}{|c|}{ No Pobre* } \\
\cline { 8 - 10 } & & Primaria & Media & Superior & & Primaria & Media & Superior \\
\hline 12 & 99,0 & 92,2 & 7,8 & & 99,9 & 90,6 & 9,4 & \\
\hline 13 & 96,8 & 51,0 & 49,0 & & 99,4 & 32,9 & 67,1 & \\
\hline 14 & 93,1 & 28,7 & 71,3 & & 97,2 & 8,9 & 91,1 & \\
\hline 15 & 85,6 & 13,8 & 86,1 & & 94,1 & 3,1 & 96,8 & \\
\hline 16 & 71,4 & 3,5 & 96,2 & 0,3 & 92,2 & 2,3 & 97,5 & 0,2 \\
\hline 17 & 67,4 & 2,6 & 96,5 & 0,9 & 87,2 & 2,3 & 94,6 & 3,1 \\
\hline 18 & 42,5 & 5,5 & 80,5 & 13,9 & 67,4 & 0,9 & 51,2 & 47,9 \\
\hline 19 & 36,2 & 1,9 & 60,1 & 38,0 & 53,3 & 1,0 & 22,4 & 76,6 \\
\hline
\end{tabular}

Fuente: Encuesta Permanente de Hogares, segundo semestre 2006.

* Terminología utilizada en la EPH.

La desigualdad por las condiciones de pobreza no constituye un fenómeno novedoso en las escuelas de los países de América Latina. Sin embargo las condiciones de pobreza en que se desarrolla la labor educativa se han agudizado en los últimos años, impactando no sólo en la situación de los estudiantes sino también en la de los propios docentes. Una investigación sobre la condición docente en cuatro países de América Latina-Argentina, Brasil, Perú y Uruguay (TENTI FANFANI, 2005)- señala cómo el cuerpo docente no 
sólo es más heterogéneo (diferencias de opiniones, valoraciones, actitudes y expectativas todas legitimas en sociedades plurales y complejas) sino que también adquiere crecientes grados de desigualdad de acuerdo con las condiciones de trabajo (incluyendo el salario percibido) y el prestigio y reconocimiento o status ocupacional asociados con la actividad docente.

En un contexto como el descripto, caracterizado por la creciente desigualdad y por el crecimiento de la pobreza, las políticas educativas de los '90 tuvieron un carácter focalizado, orientado a la reducción de la pobreza que las reformas estructurales habían contribuido a incrementar fuertemente. De este modo las políticas educativas intentaron configurarse como mecanismo de inclusión en un contexto de creciente exclusión social. Adicionalmente, se construyeron de modo sectorial, ya que se recortaron al "sector educación", suponiendo su "autonomía" con respecto al conjunto social. El carácter sectorial de la política educativa de los últimos años se puso de manifiesto tanto en la implementación de la nueva estructura del sistema educativo establecida en la Ley Federal de Educación ${ }^{10}$ como en las políticas compensatorias del Plan Social Educativo.

Respecto de la primera cuestión, la estructura académica prevista en la Ley Federal de Educación estuvo destinada, de acuerdo con los responsables ministeriales, a integrar a los alumnos históricamente excluidos del nivel medio de enseñanza, tanto a los que no accedían como a quienes desertaban en los dos primeros años del secundario. Se trató de evitar el pasaje entre uno y otro nivel del sistema educativo que la investigación mostraba como momento crítico de deserción de los adolescentes de sectores vulnerables. Si bien la implementación de la nueva estructura significó en algunas jurisdicciones una importante expansión de la matrícula, diversas investigaciones ponen de manifiesto que los supuestos beneficiarios de la modificación de la estructura son quienes han tenido mayores problemas para permanecer en el sistema.

Trabajos de investigación realizados por el Ministerio de Educación dan cuenta de que con la implementación del tercer ciclo de la EGB fue clara la intención de brindar mayor cantidad de años de escolarización a sectores que fueron excluidos del sistema educativo una vez completado el nivel primario. Los alumnos de los sectores más pobres vuelven a la escuela en la mayoría de las provincias pero sus perspectivas de permanecer no son muy promisorias considerando el modelo pedagógico del Tercer Ciclo (EGB3). Los jóvenes que ya una o varias veces abandonaron la escuela se incorporaron a las escuelas más pobres, con altos riesgos de deserción. ${ }^{11}$

Una investigación realizada por el SUTEBA (Sindicato Unificado de Trabajadores de la Educación de la Provincia de Buenos Aires) puso en evidencia que la incorporación de alumnos excluidos del sistema a la escuela resultó un hecho más formal que real, en tanto el sistema no pudo garantizarles reales posibilidades de permanencia en condiciones de realizar un verdadero aprendizaje. Los alumnos provenientes de sectores populares, supuestos beneficiarios en el discurso gubernamental de la reforma por la nueva estructura 
y la extensión de la obligatoriedad, fueron los más afectados por la improvisación con la que se implementó la puesta en marcha del octavo año (BALDUZZI; SUAREZ, 1999).

La implementación del tercer ciclo frente a la posible estrategia de abrir la escuela secundaria a los sectores excluidos, puede interpretarse como una forma de contención social frente a la fuerza desintegradora del modelo, al tiempo que se retardaba el ingreso al mercado laboral en un contexto de altos índices de desempleo (TIRAMONTI, 2001).

En cuanto a la segunda cuestión, las políticas compensatorias, en 1993 se estableció el Plan Social Educativo como componente del Plan Social del gobierno. Estuvo destinado a compensar las desigualdades a través del mejoramiento de la infraestructura escolar", la distribución de recursos, el desarrollo de acciones de capacitación docente, el estímulo a propuestas innovadoras en las escuelas y la implementación de un Programa Nacional de Becas Estudiantiles.

Desde el discurso oficial se justificó la implementación de una política focalizada señalando que ante las grandes necesidades y los recursos limitados "lo que debemos forjar es un criterio de focalización diferente al conocido en otras regiones del mundo en tiempos en que las desigualdades comienzan a agravarse. ${ }^{12}$ La orientación a grupos meta (STHAL, 1994) fue la tendencia predominante de política social estatal en los '90. La focalización de la política del PSE orientada en términos de cristalización de territorios y vínculos dependientes de los programas proveedores, rompe con la conquista de la ciudadanía y configura identidades subordinadas y dependientes a la ayuda estatal, ubicándose del lado del asistencialismo y no del derecho (DUSCHATZKY; REDONDO, 2000).

Las Becas escolares, dentro del PSE, constituyeron otra de la expresión de las políticas sociales focalizadas en el campo educativo. Una investigación sobre el Programa de Becas Estudiantiles dependiente de la Secretaría de Educación de la Ciudad de Buenos Aires (GLUZ, 2006) analiza cómo las becas configuran una estrategia de gestión de la pobreza que genera una mirada de sospecha a la vez que condescendiente hacia los becarios, estableciendo una clara frontera entre los becarios y lo nos becarios y un quiebre de los lazos sociales al interior de la escuela. A su vez alimenta el control de la vida privada de los becarios (en términos de control sobre el destino del dinero de la beca) antes que un control público sobre el destino de los recursos públicos.

Lejos de superarse la desigualdad, diversas investigaciones dan cuenta de los resultados de la implementación de estas políticas de reformas en términos de fragmentación del sistema. La investigación de Kessler (2002) reconstruye experiencias escolares fragmentadas que están socialmente estratificadas, desdibujando toda posibilidad de una experiencia educativa común.

Los trabajos de CTERA $(2004,2006)$ muestran la fragmentación del sistema a partir de la dispar implementación de la estructura académica definida por la Ley Federal de Educación y las situaciones de desigualdad y exclusión educativas que están claramente 
asociados tanto a la histórica postergación socio-económica de algunas regiones, como a los grupos sociales que han sido históricamente más afectados por los procesos de empobrecimiento. Uno de los estudios señala que si bien los niveles de escolarización entre los adolescentes y los jóvenes son relativamente altos, existe una fuerte desigualdad en el acceso, y sobre todo en la permanencia y el egreso de la educación secundaria, que se pone de manifiesto en todo el país y una importante franja de adolescentes y jóvenes están excluidos de la posibilidad de acceder a ese nivel de estudios. Sobre el conjunto de los adolescentes y jóvenes de 13 a 18 años, son un $19 \%$ quienes no concurren a la escuela, de los cuales un $0,74 \%$ nunca concurrieron a la escuela. En las regiones más postergadas del país estos porcentajes se elevan sensiblemente. En el noreste quienes no estudian son casi el treinta por ciento $(28,3 \%$,), es decir alrededor de 121.000 adolescentes y jóvenes que no concurren a la escuela. Los adolescentes y jóvenes en condición de pobreza concurren en promedio un $20 \%$ menos a la escuela que quienes no viven en esa condición (CTERA, 2006).

La fragmentación del sistema se expresa no solo a nivel interjurisdiccional sino al interior de cada una de las jurisdicciones. La investigación de Tiramonti (2004) identifica nuevos factores de estratificación que fragmentan el universo de alumnos de la escuela secundaria. El tipo de trabajo al que se aspira es uno de los aspectos que marca las fronteras entre los diferentes fragmentos. Mientras para unos constituye un medio de satisfacción y crecimiento personal y organizan sus relaciones con el trabajo desde una estética del consumo, para otros constituye el único medio posible para vivir una vida digna por lo que privilegian la ética del trabajo.

La redefinición de la educación pública y, en consecuencia también del ámbito de la educación privada, constituyó otro de los aspectos centrales en el proceso de reforma, estableciéndose una nueva conceptualización que se plasmó en la normativa sancionada durante el período. ${ }^{13}$ Este proceso contribuyó a definir un escenario favorable a los procesos de privatización en el terreno educativo.

En definitiva, las políticas educativas destinadas a incorporar a los alumnos más pobres, pusieron en evidencia las limitaciones de la lógica de focalización y contención social que orientaron dichas políticas sociales. Tal como lo señalan Lo Vuolo et al. (1999, p. 12), "las políticas que hoy se presentan como de 'lucha' contra la pobreza son tan pobres como las personas hacia quienes se dirigen." Estas políticas no se inscriben en un camino de superación del problema sino que intentan delimitarlo en un espacio social para ampliar los márgenes de tolerancia social en sociedades cada vez más desiguales.

\section{LAS PERSPECTIVAS ACTUALES}

En la actualidad, las premisas que sustentaron las políticas de reforma educativa de las últimas décadas en América Latina han comenzado a ser cuestionadas, poniendo en evidencia las limitaciones de las propuestas que buscan la reducción de la pobreza pero no la superación de la desigualdad. El caso chileno es un ejemplo elocuente de que no es 
posible democratizar los sistemas educativos sobre la base de la lógica de mercado instalada en las últimas décadas.

En nuestro país, las políticas de los últimos años se han propuesto, de acuerdo con el discurso del Ministerio Nacional "recuperar un lugar del Estado como referente político para la construcción de horizontes igualitarios para el conjunto de la sociedad" y superar las consecuencias negativas de la reforma de los ' 90 .

La derogación de la LFE -así como la actual discusión sobre la derogación y reemplazo de la Ley de Educación Superior sancionada en 1995- constituyen un quiebre respecto de las políticas de los '90, más allá de que es evidente que la normativa por si sola no va resolver los problemas de desigualdad creciente. Aún cuando la Ley de Educación Nacional mantiene algunos de los principios cuestionados en la LFE, incluye algunos avances relevantes respecto de la normativa anterior.

El Plan Social Educativo ha sido reemplazado por Programa Integral para la Igualdad Educativa. ${ }^{14}$ Este programa da cuenta de una formulación diferente a la que orientó las políticas compensatorias de los '90. En sus fundamentos se señala que "la concreción de políticas y acciones que apuntalen la construcción de la justicia social comporta una política de inclusión y de ejercicio de derechos, entre ellos, el derecho a educarse. Dentro de este concepto de promoción de la justicia social en el campo educativo, plantearnos la distribución de bienes simbólicos (culturales, sociales, pedagógicos) y el fortalecimiento de las condiciones materiales, equivale a decir que la igualdad de oportunidades educativas es una dimensión constitutiva de la igualdad social". El Programa Nacional de Inclusión Educativa se inscribe en esta misma lógica. Surge el interrogante en qué medida estos fundamentos logran plasmar en medidas de acción que modifiquen sustancialmente el modelo asistencial que orientó desde la focalización y la compensación las políticas de la década pasada. Tras una retórica que recupera la noción de igualdad y la idea de inclusión, no se ha desterrado la lógica de intervención por programas asistenciales y focalizados (GLUZ; CHIARA, 2007).

En lo que respecta al gobierno, se intenta unificar el sistema educativo a través de políticas que intentan establecer aspectos comunes para el conjunto del sistema. Por un lado la Ley de Educación Nacional (2006) intenta regular el federalismo reemplazando el Consejo Federal de Cultura y Educación por un Consejo Federal de Educación que pueda establecer, cuando lo disponga la Asamblea, resoluciones de carácter vinculante. ${ }^{14}$

Sin embargo, debemos señalar que el problema del federalismo trasciende el campo educativo. La distribución y articulación de responsabilidades, obligaciones y recursos entre el Estado Nacional y los Estados Provinciales ${ }^{15}$ para garantizar los derechos de los ciudadanos, entre ellos el derecho a la educación, se inscribe en el problema más amplio del particular federalismo en Argentina que si bien presenta diferencias con el caso de Brasil, comparte un patrón de distribución desigual de los recursos que el país produce entre las jurisdicciones que lo componen. 
La creación del Instituto Nacional de Formación Docente (INFOD), a partir de la Ley de Educación Nacional, constituye otra de las políticas tendientes a unificar el sistema, luego de la transferencia de las Instituciones de Formación Docente, a través de la "articulación de los esfuerzos de la nación y las provincias para el desarrollo, la jerarquización y la dinamización de la formación docente que incida en la mejora del sistema educativo en su conjunto". ${ }^{16}$

En lo que respecta al financiamiento del sistema, se busca compensar desigualdades entre las provincias a partir del incremento presupuestario y de la definición de un piso salarial para todos los docentes del país.

Sin embargo, perduran aún lógicas propias de los '90 en lo que respecta a los modelos de evaluación y acreditación y la definición de contenidos comunes para el conjunto del sistema.

\section{REFLEXIONES FINALES}

Las transformaciones en la regulación de las políticas educativas se enmarcan en la reforma y re-estructuración del Estado y de su administración (BARROSO, 2005). Esta reforma, como hemos visto, trajo aparejado a su vez un marcado deterioro de la situación social en América Latina en general, y en Argentina en particular.

Una característica que resalta de la implementación de las reformas educativas en el caso argentino es la abierta contradicción entre el discurso y las prácticas concretas. La imposición centralizada de mecanismos de descentralización no ha significado una delegación real de poder a los estados sub-nacionales, muchos de los cuales presentan grandes dificultades para la gestión de sus políticas por la falta de recursos, por la debilidad de su capacidad institucional y por la presencia de mecanismos clientelares en los modos históricos de construcción política.

Tras una retórica basada en la descentralización, la autonomía y la participación de la comunidad es la racionalidad neoliberal la que ha prevalecido en los procesos de reestructuración o "reinvención" del gobierno del sistema escolar y de sus instituciones.

Esta particular forma de imposición de prácticas y valores llevó a que se diera una coexistencia y articulación de nuevas formas de regulación con los viejos modos de controlar el sistema. Así, los mecanismos burocráticos tradicionales no sólo conviven con otros post-burocráticos centrados en la evaluación de resultados, sino que en muchos casos sirven para imponer su aplicación en la medida en que éstos no surgen de consensos ni de la construcción de autonomía real de los actores.

Así como en la aplicación de las reformas estructurales no se tuvieron en cuenta los altos costos sociales que generaría, tampoco en el proceso de transferencia de la responsabilidad por el servicio educativo a las jurisdicciones provinciales se consideró el impacto que tendría en la desarticulación del sistema educativo. Actualmente se ha 
perdido la idea de sistema, y se trata más bien de una totalidad constituida por fragmentos que el gobierno busca articular en torno a algunos aspectos comunes. Sin embargo, a pesar de los esfuerzos que está realizando el actual gobierno en este sentido, aún no se advierte un cambio de rumbo respecto de muchas de las reformas introducidas en los '90. Los crecientes niveles de desigualdad entre las jurisdicciones y al interior de cada una de ellas requieren de políticas social integrales en el marco de una nueva distribución de responsabilidades y atribuciones entre el gobierno central y los gobiernos provinciales, y de un proceso de redistribución social más amplio donde la nueva regulación educativa está al servicio de la democratización.

\section{Notas}

1. El Consejo Federal de Cultura y Educación fue hasta la sanción de la Ley de Educación Nacional en diciembre del 2006 el órgano responsable de planificar, coordinar, asesorar y acordar los aspectos de la política cultural y educativa. Está integrado por los Ministros o Secretarios de Educación de todas las Provincias y de la Ciudad de Buenos Aires, presidido por el Ministro de Educación de la Nación.

2. Ver BRAVSLAVSKY, C. 1983.

3. En la Provincia de Buenos Aires, principal distrito del país que abarca aproximadamente un tercio del sistema educativo argentino se intentó avanzar en un proceso de municipalización que fue resistido por la comunidad educativa y relegado luego del cambio de autoridades en el 2003.

4. El documento de Cepal/Unesco (1992) constituyó un paradigma de las propuestas de reforma en la región durante la década de los ' 90 .

5. Las reformas de primera generación tuvieron como objetivo achicar el Estado en el marco de la reconversión neoliberal de los años '70 y '80 (OSZLAK, 1999).

6. Durante el gobierno del presidente Menem, las principales normas sancionadas fueron: la Ley de Transferencia de servicios educativos de nivel medio y superior no universitario (1992); la Ley Federal de Educación (1993); la Ley de Educación Superior (1995); el Pacto Federal educativo suscripto en 1994 y convertido en Ley en 1997 y la Reforma de la cláusula constitucional acerca de las leyes de organización y de base del sistema educativo que debe sancionar el Congreso (art. 75, inc. 19 de la Constitución reformada en 1994).

Durante el gobierno del Presidente Nestor Kirchner (2003-2007) se sancionaron diversas leyes que pusieron en cuestión el modelo de los '90 e intentaron resolver problemas generados por su implementación: la Ley que establece un ciclo anual mínimo de 180 días de clase (2003); la Ley de Educación Técnico Profesional (2005); la Ley de Financiamiento Educativo (2005) y la Ley de Educación Nacional (2006) que reemplazó a la Ley Federal de Educación sancionada en 1993.

7. Tal es el caso de la extensión de la obligatoriedad, ya que hasta la fecha las políticas públicas de diferentes gobiernos no han podido garantizar los años establecidos por ley La Ley Federal de Educación (2003) estableció 10 años de educación obligatoria. La Ley de Educación Nacional (2006) extendió la obligatoriedad a 13 años hasta la finalización del nivel de educación secundaria. 
8. Nos referimos al uso del término gobernabilidad en los términos formulados por la Comisión Trilateral durante los años '70. Ver CROZIER, M. 1975.

9. Junto con el establecimiento de nuevos criterios de gestión en el funcionamiento del sistema basados en los principios de autonomía y responsabilidad individual por los resultados educativos.

10. La Ley Federal de Educación (1993) estableció una nueva estructura académica: Educación Inicial (3 a 5 años, siendo obligatorio el último año); Educación General Básica (EGB) (obligatoria a partir de los 6 años de edad con una duración de 9 años dividida en tres ciclos); Educación Polimodal (3 años de duración); Educación Superior (profesional y académica de grado en instituciones Universitarias y no universitarias) y Educación Cuaternaria.

La Ley de Educación Nacional (2006) volvió a modificar la estructura académica que comprende cuatro niveles: Educación Inicial, Educación Primaria, Educación Secundaria y Educación Superior. La Ley preve en forma transitoria dos opciones de estructura: primaria de 6 años y secundaria de 6 años o primaria de 7 años y secundaria de 5 años.

11. MINISTERIO DE EDUCACIÓN. El tercer ciclo desde la mirada docente: avances y desafíos frente a la extensión de la obligatoriedad escolar, Unidad de Investigaciones Educativas, Secretaría de Educación Básica, Buenos Aires, 2000.

MINISTERIO DE EDUCACIÓN. Implementación y localización del tercer ciclo de EGB: las prescripciones y su impacto en los actores institucionales, Unidad de Investigaciones Educativas, Secretaría de Educación Básica, Buenos Aires, 2000.

12. Una evaluación del Plan Social Educativo desde la perspectiva oficial puede verse en: ESPAÑA, S. 2003.

13. La conceptualización que subyace a la legislación sostiene que toda la educación es pública diferenciándose sólo en el tipo de gestión: estatal o privada.

14. La Ley Federal de Educación no habilitaba la toma de decisiones vinculantes por parte del Consejo Federal de Cultura y Educación, ya que las recomendaciones debían ser convalidadas por las legislaturas provinciales. Al respecto ver: IMEN, P. La Ley de Educación Nacional: una visita crítica a sus contenidos. En: IMEN, P. (en prensa).

15. El tema del federalismo incluye el Régimen de Coparticipación Federal de Impuestos. Desde la Reforma Constitucional de 1994 está pendiente la sanción de una nueva ley.

16. Información de la página del INFOD <http://www.me.gov.ar/infod/index.html>. 


\section{Referencias}

BALDUZZI, J.; SUAREZ, M. La implementación del octavo año en las escuelas bonaerenses. Cuadernos de informes e investigaciones, Buenos Aires: Suteba, n. 1, 1999. 35 p.

BARROSO, J. La autonomía de las escuelas en el contexto de cambio de los modos de regulación de las políticas y de la acción educativa: el caso portugués. Revista de Educación, Madrid, n. 333, p. 117-140, ene./abr. 2004.

BARROSO, J. O Estado, a educação e a regulação das políticas públicas. Educação \& Sociedade, Campinas, v. 26, n. 92, p. 725-751, out. 2005.

BOLIVAR, A. El lugar del centro escolar en la política curricular actual. Más allá de la reestructuración y de la descentralización. En: PEREYRA, M. A. et al. (Ed.) Globalización y descentralización de los sistemas educativos. Barcelona: Pomares-Corredor, 1996. p. 237-266.

BOLIVAR, A. La autonomía de los centros escolares en España: entre declaraciones discursivas y prácticas sobrerreguladas. Revista de Educación, Madrid, n. 333, p. 91-116, ene./abr. 2004.

BRASLAVSKY, C. Estado, burocracia y políticas educativas. En: TEDESCO, J. C.; BRASLAVSKY, C.; CARCIOFI, F. El proyecto educativo autoritario: Argentina 1976-1983. Buenos Aires: Flacso, 1983.

BRAVO, H. F. La descentralización educacional. Sobre la transferencia de establecimientos. Buenos Aires: Ceal, 1994.

CAVAROZZI, M. Autoritarismo y democracia, 1955-1983. Buenos Aires: Centro Editor de América Latina. 1992.

CEPAL-UNESCO. Educación y conocimiento: eje de la transformación productiva con equidad. Santiago de Chile: Cepal y Oficina Regional de Educación de la Unesco para América Latina y el Caribe, 1992.

CROZIER, M. et al. La crisis de la democracia. Reporte sobre la gobernabilidad de las democracias en la Comisión Trilateral. New York: University Press, 1975.

CTERA. Consecuencias de la implementación de la estructura "definida” por la Ley Federal de Educación. Los pedazos del sistema o un sistema hecho pedazos, Informes y estudios sobre la situación educativa. Buenos Aires: Instituto de Investigaciones Pedagógicas Marina Vilte, n. 2, julio 2004. 12 p.

CTERA. Desigualdad y exclusión en la educación secundaria de adolescentes y jóvenes. Informes y estudios sobre la situación educativa. Buenos Aires: Instituto de Investigaciones Pedagógicas Marina Vilte, n. 6, diciembre 2006. 46 p.

DELEUZE, G. Postdata sobre las sociedades de control. En: FERRER, C. (Comp.). El lenguaje literario. Montevideo: Nordan, 1991. p. 101-106.

DUSCHATZKY, S.; REDONDO, P. Las marcas del Plan Social Educativo o los indicios de ruptura de las políticas educativas. En: DUSCHATZKY, S. (Comp.). Tutelados y asistidos. Programas sociales, políticas públicas y subjetividad. Buenos Aires: Paidós, 2000. p. 121-185.

ECHART, M. Procesos de Reforma en Educación, Diseño, Implementación y Grupos de Interés: Evaluación de la Reforma Educativa de la Provincia de Buenos Aires. Buenos Aires: Fundación de Investigaciones Económicas Latinoamericanas, 1998. 77 p. 
ESPAÑA, S. Políticas sociales en América Latina. Mitos y realidades. Revista Iberoamericana de Educación, Madrid: OEI, n. 23, p. 51-102, mayo/ago. 2000.

FELDFEBER, M. Reforma educativa y regulación estatal. Los docentes y las paradojas de la autonomía impulsada por decreto. En: FELDFEBER, M.; OLIVEIRA, D. A. (Comp.). Políticas educativas y trabajo docente: nuevas regulaciones, ¿nuevos sujetos? Buenos Aires: Noveduc, 2006. p. 53-72.

FELDFEBER, M.; IVANIER, A. La descentralización educativa en Argentina: el proceso de transferencia de las Instituciones de Formación Docente. Revista Mexicana de Investigación Educativa, México, v. 8, n. 18, p. 421-445, mayo-ago. 2003.

FILMUS, D. Concertación educativa y gobernabilidad democrática en América Latina, Revista Iberoamericana de Educación. Madrid: OEI, n. 12, p. 11-30, sep./dic. 1996.

GARCÍA DELGADO, D. La reforma del Estado en la Argentina: de la hiperinflación al desempleo estructural Documento presentado en el I Congreso Interamericano del Clad sobre la Reforma del Estado y de la Administración Pública, Rio de Janeiro, Brasil, del 7 al 9 de noviembre de 1996.

GLUZ, N. La construcción socioeducativa del becario: la productividad simbólica de las políticas sociales en la educación media. Buenos Aires: IIPE - Unesco, 2006.

GLUZ, N.; CHIARA, M. Evaluación del Programa Integral para Igualdad Educativa (PIIE). Documento de trabajo. Momento 1: La puesta en marcha e implementación del programa y su contexto, Buenos Aires: Diniece, MECyT, 2007.

IMEN, P. La Ley de Educación Nacional: una visita crítica a sus contenidos. En: IMEN, P. Ley de educación, conocimiento y poder. Cuando lo viejo no termina de morir y lo nuevo no termina de nacer. Buenos Aires: en prensa en Ediciones del Centro Cultural de la Cooperación.

KESSLER, G. La experiencia escolar fragmentada: estudiantes y docentes en la escuela media de Buenos Aires. Buenos Aires: IIPE, 2002.

KISILEVSKY, M. Federalismo y educación: un espacio histórico de pugnas distributivas. 1998. 104 p. Tesis de Maestría, Facultad Latinoamericana de Ciencias Sociales Buenos Aires.

LO VUOLO, R. et al. La pobreza... de las politicas contra la pobreza. Madrid: Miño y Dávila Editores / Ciepp, 1999.

MEURET, D. La autonomía de los centros escolares y su regulación. Revista de Educación, Madrid, n. 333, p. 11-39, ene./abr. 2004.

NOVICK de SENÉN GONZALEZ, S. Una nueva agenda para la descentralización educativa. Revista Iberoamericana de Educación, Madrid: OEI, n. 4, p. 11-28, ene./abr. 1994.

OLIVEIRA, D. A. Regulação das políticas educacionais na América Latina e suas conseqüências para os trabalhadores docentes. Educação \& Sociedade, Campinas, v. 26, n. 92, p. 753-775, out. 2005.

OLIVEIRA, D. A. Educação básica. Gestão do trabalho e da pobreza. Rio de Janeiro: Vozes, 2000.

OSZLAK, O. De menor a mejor: el desafío de la 'segunda' reforma del Estado. Nueva Sociedad, Buenos Aires, n. 160, p. 81-100,mar./abr. 1999. 
OSZLAK, O. "Privatización y capacidad de regulación estatal: una aproximación teóricometodológica”, En: BRESSER-PEREIRA, L. C. et al. Política y gestión pública. Buenos Aires: Fondo de Cultura Económica, Clad, 2004. p. 139-193.

PAVIGLIANITI, N. Neoconservadurismo y educación. Buenos Aires: Grupo Coquena Editores, 1991.

REPETTO, F. (coordinador) Transferencia educativa hacia las provincias en los años '90. Un estudio comparado, Documento $\mathrm{n}^{\circ} 57$ del Centro de Estudios para el Desarrollo Institucional, Buenos Aires: Fundación Gobierno y Sociedad y Fundación Grupo Sophia, 2001. 104 p.

SIDICARO, R. La crisis del Estado y los actores políticos y económicos en la Argentina (1989-2001), Buenos Aires: Eudeba, Libros del Rojas, 2003.

STHAL, K. Política social en América Latina. La privatización de la crisis. Revista Nueva Sociedad, Caracas, n. 131, p. 48-71, 1994.

TENTI FANFANI, E. La condición docente: datos para el análisis comparado Argentina, Brasil, Perú y Uruguay. Buenos Aires: Siglo Veintiuno, 2005.

TENTI FANFANI, E. Nuevos problemas de gobierno de la educación en América Latina. Comentarios a las tesis de Francois Dubet. En: TENTI FANFANI, E. (Org.). Gobernabilidad de los sistemas educativos en América Latina. Buenos Aires: IIPE -Unesco, 2004. p. 45-64.

TIRAMONTI, G. (Comp.). La trama de la desigualdad. Buenos Aires: Manantial, 2004.

TIRAMONTI, G. Modernización educativa de los '90: ‘el fin de la ilusión emancipatoria?. Buenos Aires: Flacso, Temas Grupo Editorial, 2001. 


\section{Novas e velhas formas de regulação do sistema educativo na Argentina}

Resumo

Neste trabalho analisamos as politicas destinadas a regular o sistema educativo a partir de transferência de serviços educativos aos Estados subnacionais e a redefinição do papel histórico que o Estado nacional assumiu na matéria educativa. Sustentamos que as politicas promovidas pelo Estado nacional têm sido direcionadas a dar resposta à crise do modelo de regulação burocrática tradicional e a garantir a governabilidade do sistema por meio da introdução de novas tecnologias de gestão e do desenvolvimento de políticas compensatórias a serviço da redução da pobreza. Essas políticas foram propostas como a única alternativa de construçāo política viável para assentar o governo da educaçāo sobre novas bases.

Palavras-chave: Reforma Educativa. Políticas educativas. Regulação. Governo

\section{New and old form of regulation of the educational system in Argentina}

\section{Abstract}

In this essay, we analyze the policies geared to regulate the educational system on the base of the transference of educational services to sub-national states and the redefinition of the historical role that the National State has taken on with regards to educational matters. We sustain that the policies stimulated by the National State have been oriented to respond to the crisis of the model of traditional burocratic regulation and have garanteed the governability of the system through the introduction of new technologies of management and the developement of compensatory policies for the reduction of poverty. These policies have been proposed as the only alternative of viable political construction to set the foundation of the government of education on new bases.

Keywords: Educational reform. Educational Policies. Regulation. Government.

\section{De nouvelles et vieilles formes de régulation du système éducationnel en Argentine} Résumé

Dans ce travail nous analysons les politiques destinées à réguler le système éducatif à partir du transfert de services éducatifs aux états sub-nationaux et la redéfinition du rôle historique que l'État National a pris en charge en matière d'éducation. Nous soutenons que les politiques dont l'impulsion vient de l'État National ont été orientées à donner une réponse à la crise du modèle de régulation bureaucratique traditionnel et à garantir la gouvernabilité du système au travers de l'introduction de nouvelles technologies de gestion et de développement de politiques compensatoires au service de la réduction de la pauvreté. Ces politiques ont été proposées comme la seule alternative de construction politique viable pour asseoir le gouvernement de l'éducation sur de nouvelles bases.

Mots clefs : Réforme éducative. Politiques éducatives. Régulation. Gouvernement. 
